\title{
Tobacco and its impact on oral and overall health in Japan
}

\author{
Nevin Zablotsky* \\ Periodontist and Senior Consultant for Nova Southeastern University College of Osteopathic Medicine and College of Dental Medicine, USA
}

There is an old expression that always seems to bring me back to earth when I get overly optimistic about what I can accomplish in my line of tobacco cessation work.

"Man plans and God laughs." I don't think you have to be religious to get the gist of this statement, just one who understands the hubris of we human beings.

I think there finally is a consensus throughout the world that the use of tobacco products is harmful to one's health. It took legitimate scientific research, numerous peer reviewed journal publications, dedicated health advocates and effective legal teams to get there, but it seems that even the big tobacco companies now admit that perhaps the use of their products just might kill you sooner or later. Despite these dire warnings, smoking is still quite prevalent throughout the world.

In 2013, 2,528,000 Japanese adults and 33,000 children used tobacco each day. (35.5\% of men and $11.2 \%$ of women) [1] Every year 161,000 Japanese will die from tobacco related illnesses, with 2,326 men and 763 women dying weekly [1]. Despite these facts, tobacco products are still being legally sold.

There are many reasons why all dental and other health professionals in Japan, as well as throughout the world, must take the lead in working to reduce or eliminate the use of tobacco products. Some of these include:

1. Studies have shown that maternal smoking, be it passive or active, may be associated with an increased risk of cleft lips and palates in offspring [2,3].

2. There is a higher incidence of dental caries seen in those using tobacco products [4].

3. There is a causal relationship between smoking and periodontitis [4].

4. Passive cigarette smoking increases the risk of periodontal disease by over 3 times [5].

5. Smoking increases the risk of implant failure [4].

6. Leukoplakia occurs 6 times more frequently in smokers versus nonsmokers [4].

7. In 2017 , approximately 5,400 Japanese men and 3,400 women will get oral and oropharyngeal cancer [6].

8. The risk of developing oral cancer is much greater in tobacco users as 75 percent use tobacco products [4].

9. The predicted probability of high-risk HPV infection is greatest with those smoking 20 cigarettes/day [7].
10.Smoking is also the cause of one out of three of all cancer deaths, as it is known to cause cancer of the larynx, lung, bladder, pancreas, cervix, kidney, stomach, blood, liver, colon, rectum and the esophagus [4].

Given that using tobacco products is likely to compromise any dental treatment that is provided by dental healthcare providers. it behooves their profession to consider the following steps in aiding their nicotine addicted patients as well as the population in general.

"Japanese healthcare professionals can encourage their leaders to implement a tobacco control policy which will prove to be "an excellent investment in the health of Japan's population. According to the WHO, for less than $¥ 58$ per person per year Japan will be able to pay for the four 'best buys' in tobacco control policy, raising tobacco excise taxes, enforcing a comprehensive national smoke-free law and a ban on tobacco advertising and promotion, and mandating large graphic warning labels appear on tobacco product packaging. This small investment will reap enormous dividends in health and prosperity [1]."

In addition, dental professionals can assess the tobacco use of their patients, as well as their degree of nicotine addiction. Based on these findings, they can discuss the impact of their tobacco use on their oral health as well as encourage them to consider the reduction or elimination of their tobacco use. A thorough oral cancer screen should be done at dental appointments and special diligence should be followed with those patients using tobacco products.

Finally, all healthcare providers must review current peer reviewed literature in assessing any product that touts its ability to be an effective tobacco cessation aid. It will take time to be certain that these products are safe and will not create further health problems.

\section{References}

1. The Tobacco Atlas.

2. Li Z, Liu J, Ye R, Zhang L, Zheng X, Ren A (2010) Maternal passive smoking and risk of cleft lip with or without cleft palate. Epidemiology 21: 240-242. [Crossref]

3. Xuan Z, Zhongpeng Y, Yanjun G, Jiaqi D, Yuchi Z, Bing S, Chenghao L (2016) Maternal active smoking and risk of oral clefts: a meta-analysis. Oral Surg Oral Med Oral Pathol Oral Radiol 122: 680-690. [Crossref]

4. 2016: U.S Surgeon General's report.

5. Tanaka K, Miyake Y, Hanioka T, Arakawa M (2013) Active and passive smoking and prevalence of periodontal disease in young Japanese women. J Periodont Res 48: 600605. [Crossref]

Correspondence to: Nevin Zablotsky, Periodontist and Senior Consultant for Nova Southeastern University College of Osteopathic Medicine and College of Dental Medicine, USA,E-mail: nev1248@aol.com

Received: November 22, 2017; Accepted: November 30, 2017; Published: December 04, 2017 
6. Kota Katanoda, Ken-Ichi Kamo, Kumiko Saika, Tomohiro Matsuda, Akiko Shibata, et al. (2014) Short-term projection of cancer incidence in Japan using an age-period interaction model with spline smoothing. Japanese Journal of Clinical Oncology 44: 36-41.
7. Sonawane K, Suk R, Chiao EY, Chhatwal J, Qiu P, et al. (2017) Oral human papillomavirus infection: Differences in prevalence between sexes and concordance with genital human papil-lomavirus infection, NHANES 2011 to 2014. Ann Intern Med 167: 714-724. [Crossref]

Copyright: (C2017 Zablotsky N. This is an open-access article distributed under the terms of the Creative Commons Attribution License, which permits unrestricted use, distribution, and reproduction in any medium, provided the original author and source are credited. 